\title{
Differential rotation of the present and the pre-main-sequence Sun
}

\author{
M. Küker and M. Stix \\ Kiepenheuer-Institut für Sonnenphysik, Schöneckstrasse 6, 79104 Freiburg, Germany \\ e-mail: kueker@kis.uni-freiburg.de; stix@kis.uni-freiburg.de \\ Received 25 September 2000 / Accepted 9 November 2000

\begin{abstract}
We present a model for the differential rotation of the present Sun as well as a solar-type star during its pre-main-sequence evolution. The model is based on the mixing-length theory of convective heat transport and a standard solar model. The resulting rotation law is in good agreement with observations and only weakly dependent on the mixing-length parameter. For the present Sun, the normalized horizontal shear decreases with increasing rotation rate, but the total shear is roughly constant. We then follow the Sun's evolutionary track from the beginning of the contraction to the arrival on the ZAMS. While at an age of 30 Myr the total shear is very similar to that of the present Sun, it is much smaller on the Hayashi track.
\end{abstract}

Key words. hydrodynamics - Sun: rotation - stars: rotation - stars: pre-main sequence

\section{Introduction}

The differential rotation of the solar surface is a wellknown phenomenon. The rotation rate determined by Doppler measurements is about 30 percent smaller at the poles than at the equator (Snodgrass 1984), while a slightly smaller difference of 20 percent has been derived from the motion of sunspots (Howard et al. 1984; Balthasar et al. 1986). Helioseismology has revealed that this pattern is not restricted to the surface but extends throughout the whole convection zone, while the radiative core rotates rigidly with the same period as the surface at mid-latitudes (Schou et al. 1998). Surface differential rotation has also been found for a number of magnetically active stars by long-term photometry (Hall 1991; Donahue et al. 1996) and Doppler imaging (Collier Cameron et al. 2000).

Rotational shear can generate a strong toroidal magnetic field out of a weak poloidal field and is therefore the main source of magnetic field energy in the $\alpha \Omega$ dynamo which is thought to be the source of magnetic activity in solar-type main-sequence stars. In a rigidly rotating convection zone, a turbulent dynamo is still possible, but it must then be of the $\alpha^{2}$ type, which is known to prefer steady non-axisymmetric rather than oscillating axisymmetric fields (Moss \& Brandenburg 1995; Küker \& Rüdiger 1999). The knowledge of the internal rotation pattern is therefore essential in modeling stellar dynamos.

Send offprint requests to: M. Stix
Küker et al. (1993) assumed Reynolds stress as the only source of rotational shear and found a rotation pattern that remarkably well agrees with observations. The model, however, predicts an increase of the latitudinal shear with increasing rotation rate, in clear contradiction with the observational evidence. A consistent treatment of the rotation and the meridional flow yields qualitative agreement with observations but reproduces the solar rotation law only if a relatively large value of the turbulence viscosity is assumed (Rüdiger et al. 1998).

In the model of Kitchatinov \& Rüdiger (1995, KR95) this difficulty is overcome by including the convective heat transport. Kitchatinov \& Rüdiger (1993) and Kitchatinov et al. (1994) have derived a consistent mixing-length model of Reynolds stress and convective heat transport in a rotating convection zone that takes into account the effect of the Coriolis force on the convective motions. In the KR95 model, the deviation of the heat flux from spherical symmetry causes a small horizontal temperature gradient, which partly neutralizes the rotational shear as the force that drives the meridional flow. As a result, the model reproduces the original Küker et al. (1993) result with only one free parameter, the mixing-length parameter $\alpha_{\mathrm{MLT}}$.

Kitchatinov \& Rüdiger (1999) found the total shear, $\delta \Omega=\Omega_{\mathrm{eq}}-\Omega_{\text {Pole }}$,

to be only weakly dependent on the rotation period. Collier Cameron et al. (2000) have combined measurements of differential rotation for the pre-main-sequence stars RX J1508-4423, AB Dor, and PZ Tel with the sample of main-sequence stars from Donahue et al. (1996) and 
argue that the discrepancies between the Kitchatinov \& Rüdiger (1999) result and the relation between shear and rotation rate derived by Donahue et al. (1996) may be due to the mix of $\mathrm{G}$ and $\mathrm{K}$ stars in the latter paper. They suggest that $\delta \Omega$ may indeed be constant with rotation rate but vary with spectral type and derive values for $\mathrm{G}$ and $\mathrm{K}$ dwarfs that differ by a factor of three, the $\mathrm{K}$ dwarfs rotating more rigidly.

While this picture is quite appealing, the three stars mentioned above are very rapidly rotating pre-mainsequence stars and therefore differ in structure and spectral type from main-sequence stars of the same mass. One must therefore ask whether the same relation between differential rotation and spectral type holds for mainsequence and PMS stars.

In this paper, we study the differential rotation of the present Sun and a solar-type pre-main-sequence star (or the PMS Sun) at different ages. We first address the present Sun to compare the results from our model with those from previous work and observations. The model is then applied to a series of PMS models for the Sun at different ages from an evolutionary track as computed by Ahrens et al. (1992).

\section{The model}

\subsection{The transport equations}

\subsubsection{Equation of motion}

We use the mean-field formulation of hydrodynamics, i.e. apply an appropriate averaging procedure to split the velocity field into a mean and a fluctuating part,

$\boldsymbol{u}=\overline{\boldsymbol{u}}+\boldsymbol{u}^{\prime}$.

The mean velocity field, $\overline{\boldsymbol{u}}$, is then governed by the Reynolds equation,

$\rho\left[\frac{\partial \overline{\boldsymbol{u}}}{\partial t}+(\overline{\boldsymbol{u}} \cdot \nabla) \overline{\boldsymbol{u}}\right]=-\nabla \cdot(\rho Q)-\nabla p+\rho \boldsymbol{g}+\nabla \cdot \pi$,

where $Q_{i j}=\left\langle u_{i}^{\prime} u_{j}^{\prime}\right\rangle$ is the correlation tensor of the velocity fluctuations, $\boldsymbol{u}^{\prime}$. In stellar convection zones, the molecular stress tensor $\pi$ is usually about 10 orders of magnitude smaller than the Reynolds stress and can be neglected.

As the density distribution is spherically symmetric, and the gas motion is dominated by the global rotation, we assume axisymmetry for the mean velocity and temperature. The velocity field can then be described as a superposition of a rotation and a meridional flow:

$\overline{\boldsymbol{u}}=r \sin \theta \Omega \hat{\boldsymbol{\phi}}+\overline{\boldsymbol{u}}^{\mathrm{m}}$,

where $\hat{\phi}$ is the unit vector in the azimuthal direction. The azimuthal component of the Reynolds equation expresses the conservation of angular momentum:

$\frac{\partial \rho r^{2} \sin ^{2} \theta \Omega}{\partial t}+\nabla \cdot \boldsymbol{t}=0$

where

$\boldsymbol{t}=r \sin \theta\left[\rho r \sin \theta \Omega \overline{\boldsymbol{u}}^{\mathrm{m}}+\rho\left\langle u_{\phi}^{\prime} \boldsymbol{u}^{\prime}\right\rangle\right]$.
As the mass density varies with depth but not with time, mass conservation requires

$\nabla \cdot(\rho \overline{\boldsymbol{u}})=0$.

The meridional circulation can then be expressed by a stream function $A$,

$\overline{\boldsymbol{u}}^{\mathrm{m}}=\left(\frac{1}{\rho r^{2} \sin \theta} \frac{\partial A}{\partial \theta}, \frac{-1}{\rho r \sin \theta} \frac{\partial A}{\partial r}, 0\right)$.

The azimuthal component of the curl of (3) describes the meridional motion:

$$
\begin{aligned}
\frac{\partial \omega}{\partial t}= & -\left[\nabla \times \frac{1}{\rho} \nabla(\rho Q)\right]_{\phi}+r \sin \theta \frac{\partial \Omega^{2}}{\partial z} \\
& +\frac{1}{\rho^{2}}(\nabla \rho \times \nabla p)_{\phi},
\end{aligned}
$$

where $\omega=(\nabla \times \overline{\boldsymbol{u}})_{\phi}$ is the curl of the meridional flow velocity and $\partial / \partial z=\cos \theta \cdot \partial / \partial r-\sin \theta / r \cdot \partial / \partial \theta$ is the gradient along the axis of rotation.

With the expression for the specific entropy of a perfect gas,

$s=C_{\mathrm{v}} \ln \left(\frac{p}{\rho^{\gamma}}\right)$

and the relation for hydrostatic equilibrium,

$\nabla p=-\boldsymbol{g} \rho$,

the last term on the RHS of (9) may be rewritten in terms of the specific entropy,

$\frac{1}{\rho^{2}}(\nabla \rho \times \nabla p)_{\phi}=-\frac{1}{C_{\mathrm{p}} \rho}(\nabla s \times \nabla p) \approx-\frac{g}{r C_{\mathrm{p}}} \frac{\partial s}{\partial \theta}$,

where the approximation is justified though the fact that in the unperturbed (non-rotating) state the entropy is constant while the pressure is a rapidly varying function of the radius.

The stream function and the vorticity $\omega$ are related via the equation

$D A-\frac{1}{\rho} \frac{\partial \rho}{\partial r} \frac{\partial A}{\partial r}-\frac{1}{\rho r} \frac{\partial \rho}{\partial r} A=-\rho \omega$

where $D=\Delta-1 /\left(r^{2} \sin ^{2} \theta\right)$.

The correlation tensor $Q$ consists of a viscous and a non-viscous part:

$Q_{i j}=Q_{i j}^{\nu}+Q_{i j}^{\Lambda}$,

(Rüdiger 1989). The first term on the RHS of (14), the turbulence viscosity,

$Q_{i j}^{\nu}=-\mathcal{N}_{i j k l} \frac{\partial \bar{u}_{k}}{\partial x_{l}}$

has been calculated by Kitchatinov et al. (1994), while a detailed theory of the second term, the $\Lambda$ effect,

(6) $Q_{i j}^{\Lambda}=\Lambda_{i j k} \Omega_{k}$, 
(where $\boldsymbol{\Omega}=\Omega \hat{\boldsymbol{z}}$ ) has been derived by Kitchatinov \& Rüdiger (1993). The $\Lambda$ effect is proportional to the angular velocity itself rather than its gradient and thus redistributes angular momentum even in case of initially rigid rotation. All components of the correlation tensor depend on the angular velocity as well as on the convective turnover time, $\tau_{\text {corr }}$, via the Coriolis number,

$\Omega^{*}=2 \tau_{\text {corr }} \Omega$.

In the limiting case of very slow rotation, $\Omega^{*} \ll 1$, the viscous stress becomes isotropic and reduces to the wellknown expression,

$Q_{i j}^{\nu}=-\nu_{\mathrm{t}}\left(\frac{\partial \bar{u}_{i}}{\partial x_{j}}+\frac{\partial \bar{u}_{j}}{\partial x_{i}}\right)+\zeta_{\mathrm{t}} \frac{\partial \bar{u}_{k}}{\partial x_{k}} \delta_{i j}$

The $\Lambda$-effect is the source of differential rotation. In spherical polar coordinates, it is present only in two components of the correlation tensor, namely $Q_{r \phi}$ and $Q_{\theta \phi}$.

\subsubsection{Heat transport}

The convective heat transport is described by the transport equation

$\rho T \frac{\partial s}{\partial t}+\rho T \boldsymbol{u} \cdot \nabla s=-\nabla \cdot\left(\boldsymbol{F}^{\mathrm{conv}}+\boldsymbol{F}^{\mathrm{rad}}\right)+\epsilon$,

where $\epsilon$ is the source function. In case of a perfectly adiabatic stratification the entropy is constant throughout the whole convection zone. Standard mixing-length theory does not include the rotational influence on the turbulent heat transport. Kitchatinov et al. (1994) have applied the same turbulence model as in their calculation of the Reynolds stress and found

$F_{i}=\rho T \chi_{i j} \nabla_{j} s$,

where

$\chi_{i j}=\chi_{\mathrm{t}}\left(\left[\phi_{1}+\phi_{2}\right] \delta_{i j}+\left[\phi_{1}-\phi_{2}\right] \frac{\Omega_{i} \Omega_{j}}{\Omega^{2}}\right)$

is the turbulent heat conductivity tensor. The functions $\phi_{1}$ and $\phi_{2}$ are given by

$\phi_{1}\left(\Omega^{*}\right)=\frac{3}{4 \Omega^{* 2}}\left(-1+\frac{\Omega^{* 2}+1}{\Omega^{*}} \arctan \Omega^{*}\right)$,

$\phi_{2}\left(\Omega^{*}\right)=\frac{3}{2 \Omega^{*}}\left(1-\frac{\arctan \Omega^{*}}{\Omega^{*}}\right)$.

In case of very slow rotation, i.e. $\Omega^{*} \ll 1$, the heat conductivity tensor reduces to the standard mixing-length expression,

$\chi_{\mathrm{t}}=\frac{1}{3} \tau_{\text {corr }} u_{\mathrm{t}}^{2}$,

where $\tau_{\text {corr }}$ is the convective turnover time. The convection velocity, $u_{\mathrm{t}}$, is given by

$u_{\mathrm{t}}^{2}=-\frac{l^{2} g}{4 C_{\mathrm{p}}} \frac{\partial s}{\partial r}$ where

$l=\alpha_{\mathrm{MLT}} H_{\mathrm{p}}$

is the mixing length and

$H_{\mathrm{p}}=-\frac{p}{\frac{\partial p}{\partial r}}$

the pressure scale height. The convection velocity and the mixing length together determine the convective turnover time through the relation

$\tau_{\text {corr }}=\frac{l}{u_{\mathrm{t}}}$.

The eddy viscosity and heat conductivity coefficients are thus given by

$\nu_{\mathrm{t}}=-\frac{\tau_{\mathrm{corr}} g \alpha_{\mathrm{MLT}}^{2} H_{\mathrm{p}}^{2}}{15 C_{\mathrm{p}}} \frac{\partial s}{\partial r}$,

$\chi_{\mathrm{t}}=-\frac{\tau_{\mathrm{corr}} g \alpha_{\mathrm{MLT}}^{2} H_{\mathrm{p}}^{2}}{12 C_{\mathrm{p}}} \frac{\partial s}{\partial r}$.

\subsection{Model of the convection zone}

KR95 used a simplified model of the convection zone which is derived from the full model by solving the equations

$\frac{\mathrm{d} T}{\mathrm{~d} r}=-\frac{g(r)}{C_{\mathrm{p}}}, \quad \frac{\mathrm{d} g}{\mathrm{~d} r}=-2 \frac{g}{r}+4 \pi G \rho, \quad \rho=\rho_{\mathrm{e}}\left(\frac{T}{T_{\mathrm{e}}}\right)^{\frac{1}{\gamma-1}}$

from a starting point $x_{\mathrm{e}}$, where the reference values $g_{\mathrm{e}}, \rho_{\mathrm{e}}$ and $T_{\mathrm{e}}$ are taken from a standard solar model. Together with the opacity law, $\kappa=0.34\left(1+610^{24} \rho T^{-7 / 2}\right) \quad$ (in cgs), this gives a stratification quite close to that of the model the reference values were taken from. This, however, does not work in case of the PMS models, where the KR95 model fails to reproduce the correct depth of the convection zone.

We have therefore used the above model for testing purposes only and instead take the stratifications of density, temperature, and luminosity directly from the solar model by Ahrens et al. (1992). Moreover, we use the opacity tables (Weiss et al. 1990) that were used to compute the solar model rather than a simple power law.

During the early stages of solar evolution when the Sun is still on or close to the Hayashi track the total luminosity is not constant with radius throughout the whole convection zone because gravitational energy is released everywhere in the solar volume. In these cases we also include a source function in the heat transport equation.

\subsection{Boundary conditions}

Our model does not include an atmosphere. Instead, the model ends $0.02-0.05 R_{\odot}$ below the photosphere. Depending on the state under consideration, the lower boundary is located either at the bottom of the convection zone or, in those cases where the Sun is still fully convective, at $0.1 R_{\odot}$. 
We do not consider convective overshooting at the boundaries. Below the convection zone, linear and angular momentum is transported by molecular viscosity, which is about ten orders of magnitude smaller than the Reynolds stress, while the gas density in the atmosphere is so small that no significant stress can be maintained on the convection zone. We therefore require that both the upper and lower boundaries be stress-free,

$Q_{\mathrm{r} \phi}=Q_{\mathrm{r} \theta}=0$.

As boundary condition for the heat flux we require that the total flux through the boundaries is equal to the total luminosity at that particular radius:

$F_{\mathrm{r}}^{\mathrm{tot}}(r)=\frac{L(r)}{4 \pi r^{2}}$

where $r=r_{\mathrm{i}}, r_{\mathrm{e}}$ are the inner and outer radii of the convection zone. In the convection zone of the present Sun, there is no source of energy and hence the total heat flux is constant with radius:

$F_{\mathrm{r}}^{\mathrm{tot}}\left(r_{\mathrm{i}}\right) r_{\mathrm{i}}^{2}=F_{\mathrm{r}}^{\mathrm{tot}}\left(r_{\mathrm{e}}\right) r_{\mathrm{e}}^{2}$.

In general, the luminosity may vary with radius and (33) does not hold.

With the boundary conditions (32), $F_{\mathrm{r}}$ and hence the gradient of the entropy does not vary with latitude on the boundaries. The entropy itself, however, does.

We have also tried the outer boundary condition of KR95,

$F_{\mathrm{r}}^{\mathrm{tot}}\left(r_{\mathrm{e}}\right)=\frac{L}{4 \pi r_{\mathrm{e}}^{2}}\left(1+4 \frac{T \delta s}{C_{\mathrm{p}} T_{\mathrm{eff}}}\right)$

and found only small differences. However, (34) does not ensure that the luminosity is constant with radius and the solutions show a slow drift in the total entropy while with (32) a stationary state is reached after a few diffusion times.

\subsection{Numerical scheme}

Equations (5), (9), and (19) for the unknown functions $\Omega, \omega$, and $s$ are solved with a time-dependent explicit finite-difference scheme in spherical polar coordinates, while Eq. (13) is reduced to a set of ordinary differential equations by expansion in terms of spherical harmonics. The code is an extension of that used by Küker \& Rüdiger (1997), the main modification being the addition of Eq. (19). The mesh size $N_{\mathrm{r}} \times N_{\theta}$ varies from $100 \times 80$ for the present Sun at moderate rotation rates to $300 \times 120$ for the pre-main-sequence Sun. The expansion in terms of spherical harmonics is truncated after $l=10$ for the present Sun, while for the pre-main-sequence models the truncation level might increase up to $l=40$. The code was checked by reproducing some of the results of KR95.

\section{Results}

We are particularly interested in the normalized equatorpole difference of the rotation rate,

$\delta \tilde{\Omega}=\frac{\delta \Omega}{\Omega_{\mathrm{eq}}}$

\subsection{Present Sun: Variation of the rotation period}

Figure 1 shows the result for the present Sun with a value of $5 / 3$ for the mixing-length parameter for rotation periods ranging from $56 \mathrm{~d}$ to $7 \mathrm{~d}$.

At a rotation period of $56 \mathrm{~d}$ we find a value of 33 percent for $\delta \tilde{\Omega}$. The meridional flow shows one cell per hemisphere with the gas motion being equatorward at the surface and poleward at the bottom of the convection zone. The maximum flow velocity is $9.2 \mathrm{~m} / \mathrm{s}$ at the surface and $0.3 \mathrm{~m} / \mathrm{s}$ at the bottom of the convection zone.

The case $P=28 \mathrm{~d}$ corresponds to the observed solar rotation rate. The equator rotates about 20 percent faster than the poles at the top of the convection zone. The meridional flow pattern consists of two cells per hemisphere, with the flow directed towards the equator at both the bottom and the top of the convection zone and poleward at intermediate depths. The maximum speed of the horizontal motion is $5.4 \mathrm{~m} / \mathrm{s}$ at the surface and $2 \mathrm{~m} / \mathrm{s}$ at the bottom of the convection zone.

For a rotation period of $14 \mathrm{~d} \delta \tilde{\Omega}$ decreases to 10 percent. There are still two flow cells per hemisphere, but the outer cell is restricted to a shallow surface layer. The surface flow is still poleward with an amplitude of $3.8 \mathrm{~m} / \mathrm{s}$. At the bottom of the convection zone the flow reaches a speed of $2.8 \mathrm{~m} / \mathrm{s}$.

Decreasing the rotation period to $7 \mathrm{~d}$ leads to a further reduction of $\delta \tilde{\Omega}$ to less than five percent. The meridional flow is now directed poleward at the top and equatorward at the bottom of the convection zone, with flow speeds of $2.7 \mathrm{~m} / \mathrm{s}$ at the top and $3.6 \mathrm{~m} / \mathrm{s}$ at the bottom of the convection zone.

\subsection{Present Sun: Variation of the mixing-length parameter}

We now keep the rotation period fixed at $28 \mathrm{~d}$ and vary $\alpha_{\mathrm{MLT}}$ to check how strongly our results depend on this parameter. The results are shown in Fig. 2.

For a small value of 0.5 the surface rotation at the equator is 34 percent faster than at the poles. There is one flow cell per hemisphere with an equatorward directed surface flow of up to $5 \mathrm{~m} / \mathrm{s}$ and a poleward flow of $1.2 \mathrm{~m} / \mathrm{s}$ at the bottom of the convection zone.

A value of 1.0 for $\alpha_{\text {MLT }}$ yields a value of 24 percent for $\delta \tilde{\Omega}$. The surface flow is directed towards the equator and reaches maximum speed of $5.3 \mathrm{~m} / \mathrm{s}$. At the bottom, the flow is poleward with an amplitude of $0.8 \mathrm{~m} / \mathrm{s}$.

At $\alpha_{\mathrm{MLT}}=1.3$ the difference of the surface rotation rates has decreased to 22 percent. The surface flow is still 

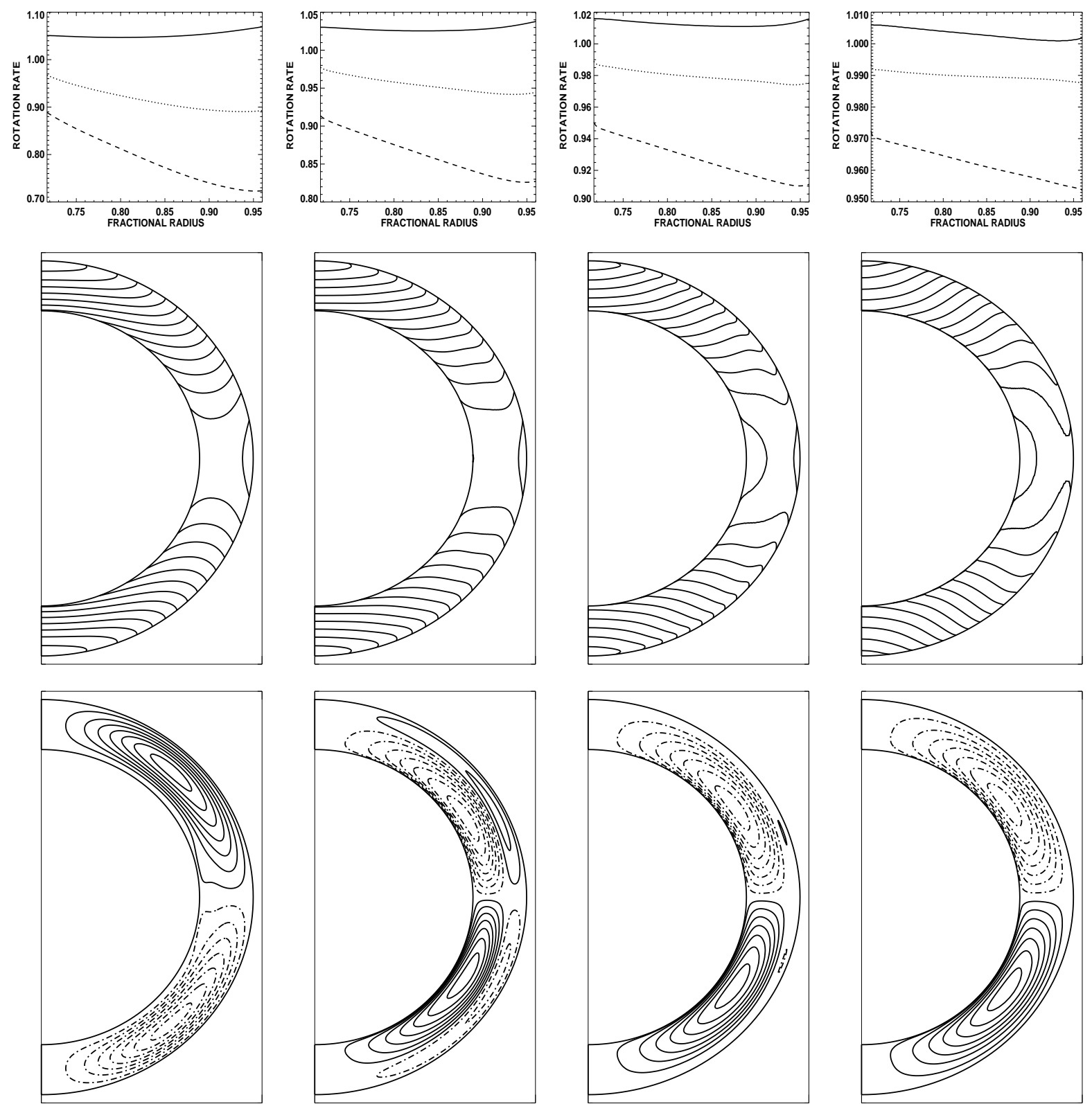

Fig. 1. The rotation and meridional flow patterns for the Sun at an age of 4.62 Gyr with $\alpha_{\mathrm{MLT}}=5 / 3$ for rotation periods $P_{\text {rot }}=56 \mathrm{~d}, 28 \mathrm{~d}, 14 \mathrm{~d}, 7 \mathrm{~d}$ (from left to right). Top row: the normalized rotation rate at the equator (solid lines), $45^{\circ}$ latitude (dotted), and the poles (dashed). Middle row: isocontour plot of the rotation rate. Bottom row: isocontours of the stream function. Dash-dotted lines denote counterclockwise circulation

equatorward with speeds up to $5.4 \mathrm{~m} / \mathrm{s}$. The bottom flow is equatorward at high and poleward at low latitudes with speeds up to $0.4 \mathrm{~m} / \mathrm{s}$.

For a mixing length parameter as large as $2.5 \delta \tilde{\Omega}$ still reaches a value of 20 percent. The flow pattern is dominated by one large cell per hemisphere in the bulk of the convection zone plus a very shallow surface layer with clockwise flow in the northern and counter-clockwise flow in the southern hemisphere (not visible in the plot). The flow reaches a maximum speed of $3.3 \mathrm{~m} / \mathrm{s}$ at the surface and $8.6 \mathrm{~m} / \mathrm{s}$ at the bottom of the convection zone.

\subsection{The young Sun}

We now fix the rotation period at $28 \mathrm{~d}$ and the mixinglength parameter at a value of $5 / 3$ and vary the age. Figure 3 shows the results for the first model of our sequence, at $L \approx 7 L_{\odot}$ on the Hayashi line of the Hertzsprung-Russell diagram, and for models with ages of 3,10 , and $31 \mathrm{Myr}$, relative to that first model, which we call the $t=0$ model.

The first model of the sequence is still fully convective. (The small core has numerical reasons.) With a value of only 2.7 percent for $\delta \tilde{\Omega}$ the rotation is almost rigid. The meridional flow is dominated by one large cell per hemisphere, but in a shallow layer at the top there is a second 

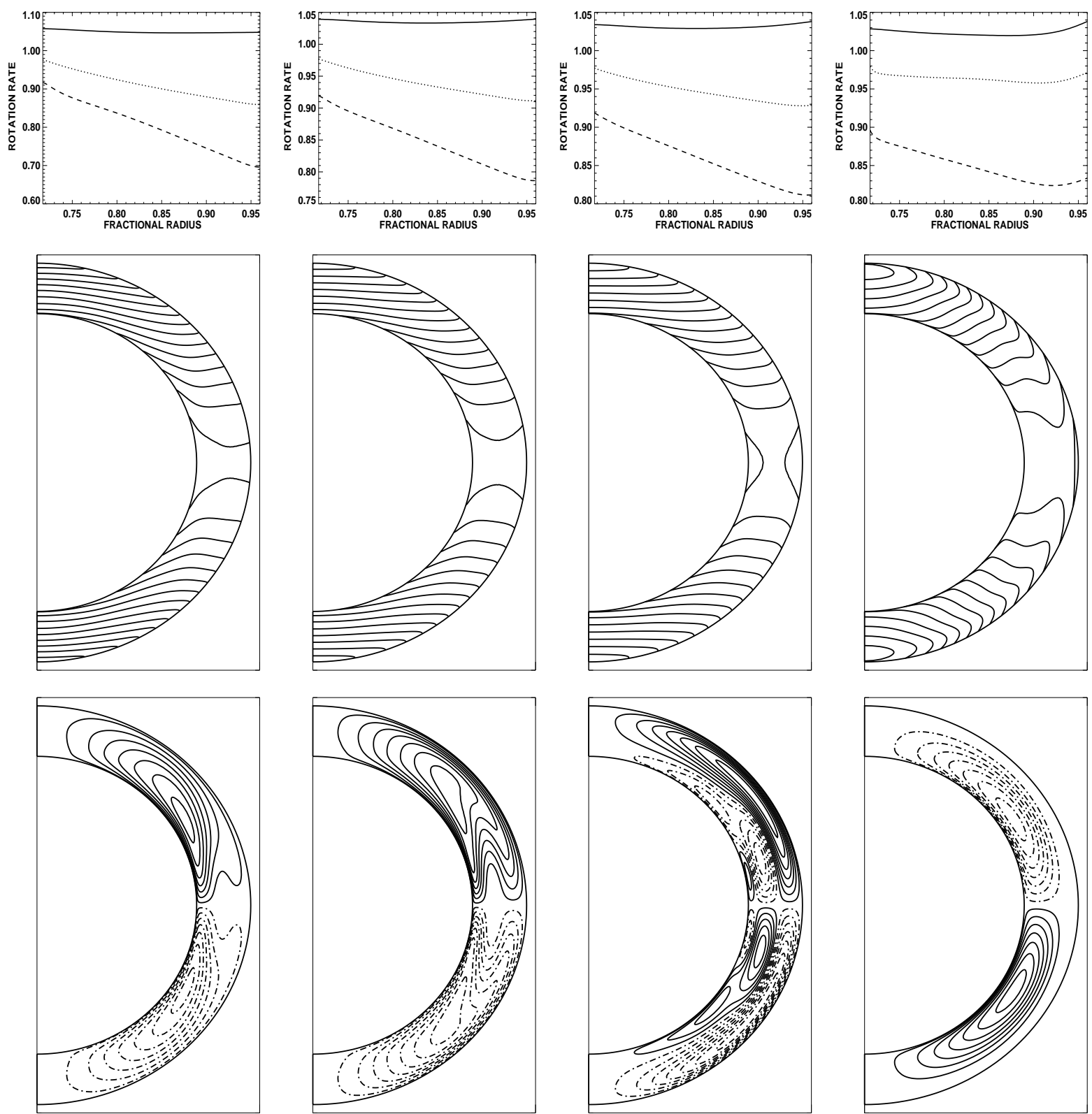

Fig. 2. Same as Fig. 1, but for fixed a rotation period of $28 \mathrm{~d}$ and a varying value of the mixing-length parameter. From left to right: $\alpha_{\mathrm{MLT}}=0.5,1.0,1.3,2.5$

cell with the opposite direction (not visible in the plot). The gas moves equatorward at the surface and close to the center. The flow reaches a speed of $11.5 \mathrm{~m} / \mathrm{s}$ at the surface and $14.1 \mathrm{~m} / \mathrm{s}$ close to the center.

After $3 \mathrm{Myr}$, at a luminosity of $0.9 L_{\odot}$, a core has developed and fills $1 / 3$ of the solar radius. The differential rotation now assumes a value $\delta \tilde{\Omega}=3.2$ percent. The surface flow is directed equatorward and reaches a speed of $6.7 \mathrm{~m} / \mathrm{s}$. At the bottom of the convection zone the gas moves equatorward, too, with a maximum speed of $3.7 \mathrm{~m} / \mathrm{s}$.

At an age of $10 \mathrm{Myr}$, the Sun has just left the Hayashi line. Its luminosity is less than one-half of the present value, and the radiative core has grown to 53 percent of the radius. The rotational shear reaches 3.9 percent. The surface flow is directed equatorward with a maximum speed of $1.2 \mathrm{~m} / \mathrm{s}$. At the bottom, the gas moves equatorward with speed up to $1.9 \mathrm{~m} / \mathrm{s}$.

The last model of Fig. 3 has an age of 31 Myr. It lies on the small loop in the HRD that the Sun went through before settling at the zero-age main sequence. The luminosity is again $\approx 0.9 L_{\odot}$, but the convection zone has retreated from the inner 70 percent of the solar radius. Now $\delta \tilde{\Omega}$ assumes a value of 18.6 percent. The maximum flow velocity is $6.6 \mathrm{~m} / \mathrm{s}$ at the top and $2.9 \mathrm{~m} / \mathrm{s}$ at the bottom, both directed equatorward.

\section{Discussion}

For the present Sun, rotating with a period of $28 \mathrm{~d}$, the results from our model agree quite well with the observations, although some discrepancies remain to be explained. 

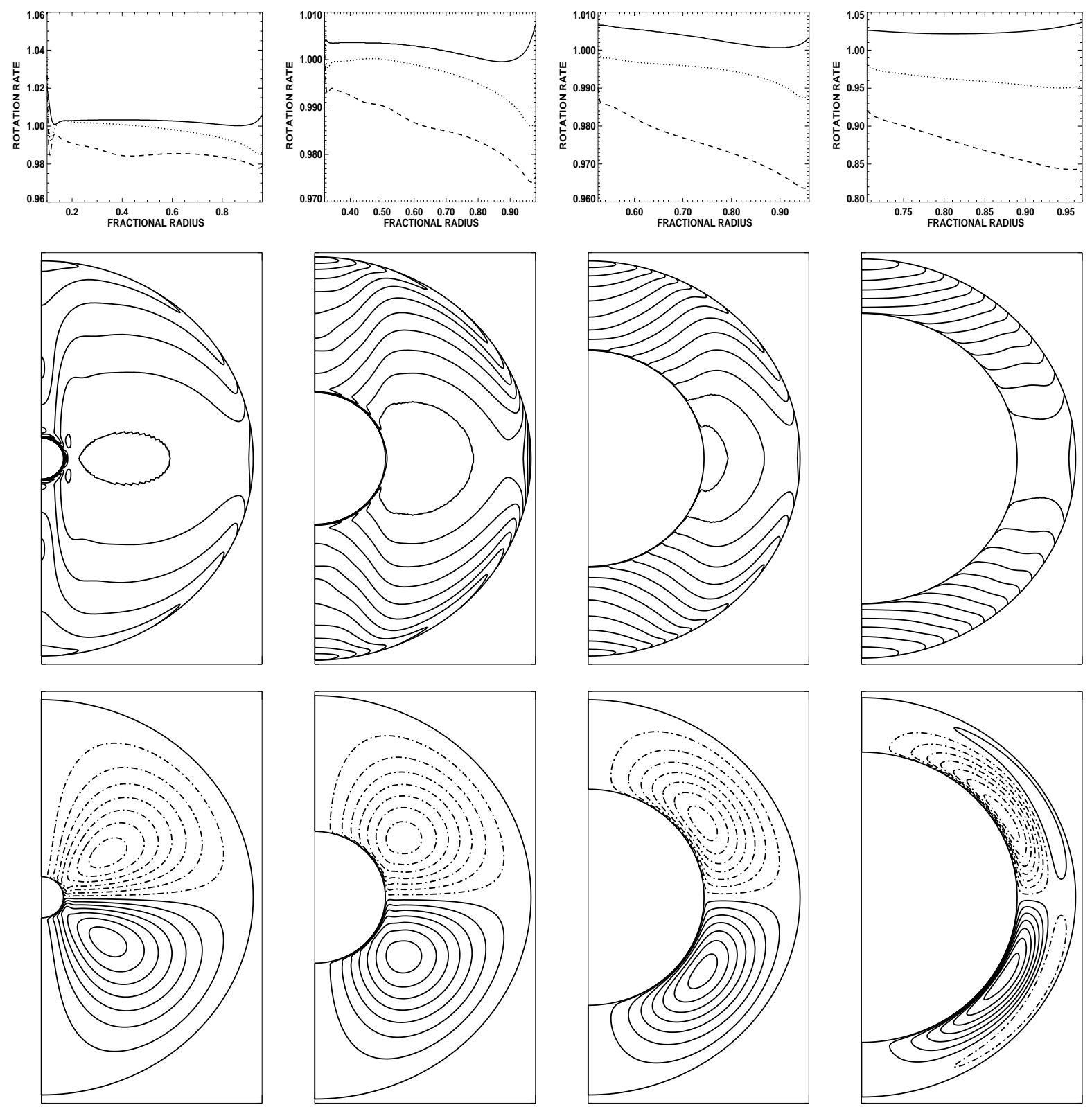

Fig. 3. Same as Fig. 1, but for fixed rotation period of 28 d, mixing-length parameter fixed to a value of $5 / 3$, and varying age. From left to right: $t=0,3 \mathrm{Myr}, 10 \mathrm{Myr}, 31 \mathrm{Myr}$. Note that the total radius varies with age

Observations show an increase of the rotation rate with increasing depth in the top layers of the convection zone, while our model produces a decrease. In the model, the sign of the vertical rotational shear at the surface is a consequence of the sign of the $\Lambda$ term in the Reynolds stress. For small values of the Coriolis number, the angular momentum transport is outward and therefore $\partial \Omega / \partial r>0$.

The reason for this behavior is that in the strictly local mixing-length model we use, the convective turnover time and hence the Coriolis number drops to zero at the outer boundary. There is therefore always a thin surface layer where $\Omega^{*}<1$, and hence the rotation rate must always increase with radius, i.e. decrease with depth close to the photosphere. This feature does not significantly change the global rotation pattern, but it drives the clockwise circulation of the meridional flow in the surface layer and the equatorward surface flow, which also contradicts the observations.

For the model of the present Sun, the total latitudinal shear, $\delta \Omega$, is roughly the same for the rotation periods of $28 \mathrm{~d}, 14 \mathrm{~d}$, and $7 \mathrm{~d}$, but decreases for slow rotation, as the result for $P=56 \mathrm{~d}$ shows. This indicates that $\delta \Omega$ depends on stellar structure rather than on the rotation rate, as predicted by earlier models (Kitchatinov \& Rüdiger 1993; Rüdiger et al. 1998).

The dependence of the rotation pattern on the mixinglength parameter proves to be moderate, although the $\Lambda$ effect is proportional to $\alpha_{\mathrm{MLT}}^{2}$. Small values of $\alpha_{\mathrm{MLT}}$ yield a disc-shaped pattern, i.e. the rotation rate is constant with distance from the rotation axis, while for large values the rotation rate is constant with the distance from the solar center. The meridional flow, on the other hand, 
completely changes as $\alpha_{\mathrm{MLT}}$ is increased from 0.5 to 2.5 . Note that for small values of the mixing-length parameter the flow structure at low latitudes differs from that of the remainder part of the convection zone.

A comparison of the results for the pre-main-sequence models shows that the total latitudinal shear is close to that of the present Sun for the 31 Myr model, but much smaller for ages up to 10 Myr. The shear between the equator and the poles thus builds up between the departure from the Hayashi track and the arrival on the main sequence. With a more realistic choice of $7 \mathrm{~d}$ or even less for the rotation period the normalized shear, $\delta \tilde{\Omega}$, would, however, still be rather small at $31 \mathrm{Myr}$, because the total shear does not appear to depend on the rotation rate and fast rotation thus means a small value of $\delta \tilde{\Omega}$. When the solar rotation slows down on the main sequence, the total shear then finally shows up as relative shear between the equator and the poles.

\section{Conclusions}

In this paper, we have studied the differential rotation of the Sun during its pre-main-sequence evolution. As a first step, we have applied a model of the turbulent transport of heat and angular momentum proposed by KR95 to a more realistic model of the solar convection zone and confirmed their findings. The solar rotation pattern as observed by helioseismology is in general well reproduced, but there is a significant deviation in the uppermost layer, where the convective turnover time is short and the density scale height is small.

The discrepancy could probably be removed through a non-local definition of the convective turnover time. However, the observed surface pattern has a characteristic time scale of several minutes only and should thus indeed hardly be affected by the global rotation as indicated by the small value of the Coriolis number. Another possible explanation could be that the stress-free boundary condition is unrealistic, but any other boundary condition would imply the introduction of external torques. The only significant torque, however, is magnetic braking by the solar wind, which acts on a time scale of Gyr rather than years, as the internal stress does. We therefore conclude that magnetic stresses are negligible in the current context. This leaves us with the possibility that the Kitchatinov \& Rüdiger (1993) theory of the $\Lambda$ effect might yield incorrect results for the uppermost layers of the solar convection zone, where the density scale height varies rapidly with depth, and where the Coriolis number is small due to the small size of the convection cells.

We have then followed the evolution of the solar rotation pattern along the evolutionary track from the fully convective state at the Hayashi line to the arrival on the main sequence and found that the rotational shear observed on the surface of the present Sun builds up when the Sun moves from the Hayashi track to the main sequence.
Between the departure from the Hayashi track and the arrival on the main sequence the effective temperature increases from $4200 \mathrm{~K}$ to $5600 \mathrm{~K}$, i.e. the spectral type changes from $\mathrm{K}$ to $\mathrm{G}$. Our finding that the shear is much smaller on the Hayashi track than on the main sequence is therefore in agreement with the conclusion of Collier Cameron et al. (2000) that differential rotation depends on spectral type rather than rotation rate.

The small value of $\delta \Omega$ on the Hayashi track confirms the result of Küker \& Rüdiger (1997), who found essentially rigid rotation for a $1.5 M_{\odot}$ PMS star and supports their conclusion that the magnetic activity of $\mathrm{T}$ Tauri stars is most likely due to an $\alpha^{2}$ dynamo. As this type of dynamo prefers non-axisymmetric field configurations while $\alpha \Omega$-type dynamos generate axisymmetric fields, the change of the dynamo from one type to the other due to the retreat of the convection zone should cause a change of the field geometry, which might be quite important for the angular momentum evolution of the Sun. In this context one should also note that, given the total shear is a function of the spectral type only, the level of magnetic activity depends on the stellar rotation rate only through the turbulent electromotive force, because the main field generation term of the $\alpha \Omega$ dynamo, $\nabla \Omega$, does not depend on the rotation rate.

\section{References}

Ahrens, B., Stix, M., \& Thorn, M. 1992, A\&A, 264, 673

Balthasar, H., Vázquez, M., \& Wöhl, H. 1986, A\&A, 155, 87

Collier Cameron, A., Barnes, J. R., \& Kitchatinov, L. L. 2000, in Proceedings of the Eleventh Cambridge Workshop on Cool Stars, Stellar Systems, and the Sun, ed. R. J. Garcia Lopez, R. Rebolo, \& M. R. Zapatero Osorio, ASP Conf. Ser., in press

Donahue, R. A., Saar, S. H., \& Baliunas, S. L. 1996, ApJ, 466, 384

Hall, D. 1991, in The Sun and Cool Stars: activity, magnetism, dynamos, ed. I. Tuominen, D. Moss, \& G. Rüdiger, Springer Lecture Notes in Physics, 380

Howard, R., Gilman, P. A., \& Gilman, P. I. 1984, ApJ, 283, 373

Kitchatinov, L. L., \& Rüdiger, G. 1993, A\&A, 276, 96

Kitchatinov, L. L., Pipin V. V., \& Rüdiger, G. 1994, Astron. Nachr., 315, 157

Kitchatinov, L. L., \& Rüdiger, G. 1995, A\&A, 299, 446 (KR95)

Kitchatinov, L. L., \& Rüdiger, G. 1999, A\&A, 344, 911

Küker, M., Rüdiger, G., \& Kitchatinov, L. L. 1993, A\&A, 279, L1

Küker, M., \& Rüdiger, G. 1997, A\&A, 328, 253

Küker, M., \& Rüdiger, G. 1999, A\&A, 346, 922

Moss D., \& Brandenburg A. 1995, Geophys. Astrophys. Fluid Dyn., 80, 229

Rüdiger, G. 1989, Differential rotation and stellar convection: Sun and solar-type stars (Gordon \& Breach, New York)

Rüdiger, G., Rekowski, B. von, Donahue, R. A., \& Baliunas, S. L. 1998, ApJ, 494, 691

Schou, J., Antia, H. M., Basu, S., et al. 1998, ApJ, 505, 390

Snodgrass, H. B. 1984, Solar Phys., 94, 13

Weiss, A., Keady, J. J., \& Magee, N. H. Jr. 1990, Atom. Data Nucl. Data Tables, 45, 209 